\title{
Reconstruction of Humeral Head Defect in Locked Posterior Dislocation Shoulder. A Case Series of Nine Patients
}

\author{
Said K. Abdel-Hameed1, Abdel-Khalek A. Ibrahem Alzalabany1, Mohamed A. Abdel-Aal2*, \\ Abdel-Aleem Soltan ${ }^{1}$ \\ ${ }^{1}$ Orthopedic Surgery, Faculty of Medicine for Girls, Al-Azhar University, Cairo, Egypt \\ ${ }^{2}$ Orthopedic Surgery, El-Bakry General Hospital, Ministry of Health, Cairo, Egypt \\ Email: ${ }^{*}$ btmnail2010@hotmail.com
}

Received 4 January 2015; accepted 13 February 2015; published 17 February 2015

Copyright ( 2015 by authors and Scientific Research Publishing Inc.

This work is licensed under the Creative Commons Attribution International License (CC BY).

http://creativecommons.org/licenses/by/4.0/

(c) (i) Open Access

\begin{abstract}
Locked posterior dislocation shoulder is uncommon and frequently missed injury. It account for $\mathbf{2 \%}$ - 4\% of all shoulder dislocations. It is commonly associated with osseous defects in humeral head articular surface known as reverse Hill-Sachs lesion. Numerous surgical procedures invented to repair this defect with variable outcomes but evidence based management strategies are lacking. Among these procedures are: transfer of lesser tuberosity or subscapularis tendon, rotational osteotomy of humerus, osteochondral grafts. Salvage procedure as hemiarthroplasty or total shoulder arthroplasty used in huge non-constructable defect or very old neglected dislocation. In our case series, we treated 9 cases ( 2 females) of locked posterior shoulder dislocation with anteromedial humeral head defects ranging between $30 \%-50 \%$ of head size. Open reduction of dislocation followed by transfer of the lesser tuberosity together with subscapularis tendon for reconstruction of the humeral head defect. The transfer was fixed with Ethibond suture size 5-0 (Ethicon, Inc. Somerville, New Jersy). The mean follow-up period was 14.5 months (range, 12 - 25 months). Seven cases had no pain or restriction of activities of daily living. No patient had symptoms of instability of the shoulder. According to UCLA Shoulder rating scale, there were 3 cases rated excellent, 4 cases rated good, one case rated fair and one case rated poor. It is concluded that reconstruction of the humeral head defect provides good pain relief, stability and function for patients with a locked posterior dislocation where the defect involves between $\mathbf{3 0 \%}-\mathbf{5 0 \%}$ of the articular surface circumference. Our technique is simple, cheap and there is no need for second operation for hardware removal.
\end{abstract}

${ }^{*}$ Corresponding author.

How to cite this paper: Abdel-Hameed, S.K., et al. (2015) Reconstruction of Humeral Head Defect in Locked Posterior Dislocation Shoulder. A Case Series of Nine Patients. Open Journal of Orthopedics, 5, 25-33.

http://dx.doi.org/10.4236/ojo.2015.52004 


\section{Keywords}

\section{Locked Posterior Shoulder Dislocation, Reverse Hill-Sachs Lesion, Anatomic Reconstruction}

\section{Introduction}

Posterior dislocations of the humeral head are rare, comprising only $2 \%-4 \%$ of shoulder dislocations [1] [2]. They mostly occur secondary to violent muscle contractions associated with seizures, electric shock or (sports) trauma [3]. Half of these dislocations are associated with an impression fracture of the anteromedial aspect of the humeral head or "reverse Hill-Sachs lesion" [3] [4].

There are no evidence-based management strategies concerning the humeral head impaction, but different surgical options described such as a transfer of the subscapularis tendon or lesser tuberosity into the defect [5] [6] and rotational osteotomy of the proximal humeral head [7]. Other procedures include cancellous bone graft either autogenic or allogenic [8] and arthroplasty [9].

The purpose of this study is to report the results of open reduction and reconstruction of the humeral head defect through transfer of subscapularis tendon or lesser tuberosity and in both conditions we fix the transfer with size [5] Ethibond suture (Ethicon, inc. Somerville, New Jersey) instead of using screws or stables for fixation. It is concluded that patients with locked posterior dislocation where an osseous defect involves between $30 \%-50 \%$ of articular surface, reconstruction of the defect provides good pain relief, range of motion, stability and patient satisfaction.

\section{Patients and Methods}

This was a prospective case series study of 9 patients with neglected locked posterior dislocation of the shoulder and reverse Hill-Sachs lesion treated and followed between March 2009 and October 2012. The mean follow-up period was 18 months (range, 14 - 25 months). There were 7 males and 2 females, right shoulder affected in 6 patients. The mean age of the patients was 29.50 (range, 22 - 46). Six patients had been correctly diagnosed, while 3 patients received a program of physiotherapy owing to wrong diagnosis as frozen shoulder. The chief complaint of all patients was pain and limited external rotation. Causative trauma was fall from height in 4 patients; seizer due to epileptic fits in 2 patients, electric shock in one case and in 2 cases the exact cause could not be detected. All patients underwent a thorough standardized physical and neurological examination before surgery. The average forward flexion was $90^{\circ}$, internal rotation to L5, with no external rotation. The patients kept their forearm against their chest somewhat comfortably. Pre-operative anteroposterior view might be misleading (Figure 1). Axillary or scapular views would confirm the diagnosis (Figure 2). Preoperative CT scans were obtained for all patients, and mean humeral head defect was 40\% (range, 35\% - 45\%) (Figure 3 and Figure 4).

The characteristics of the cases are shown in Table 1.

\section{Surgical Technique}

All patients had been operated-upon under general anesthesia and utilizing beach-chair position with the upper body out of the operating table allowing full extension of arm. Delto-pectoral approach was used in all cases with preservation of the cephalic vein (Figure 5). The superior $2 \mathrm{~cm}$ of the pectoralis major were released to provide exposure to the inferior aspect of the subscapularis and the anterior circumflex vessels. Then the arm was externally rotated to further expose the boundaries of the subscapularis and the anterior circumflex vessels and facilitating the release of the capsule from the humerus to the six o'clock position on the humerus. The lesser tuberosity was osteotomized with subscapularis tendon and was elevated to expose the humeral head. After releasing the posterior capsule and all the soft tissue a small Cobb elevator was inserted through the rotator interval to the posterior part of the glenoid rim and reduction of the dislocation was accomplished with lateral distraction and external rotation (Figure 6). Good irrigation of the joint and proper debridment of bony and cartilaginous debris was done. The lesser tuberosity was transferred into the defect and with the help of a Giant Needle (size $2.5 \mathrm{~mm}$ ) and 2 - 3 transosseous horizontal sutures with Ethibond 5-0 passed from lesser tuberosity to the humeral head, then retrieved and tied lateral to the bicipital groove (Figure 7). Stability of the shoulder and the 


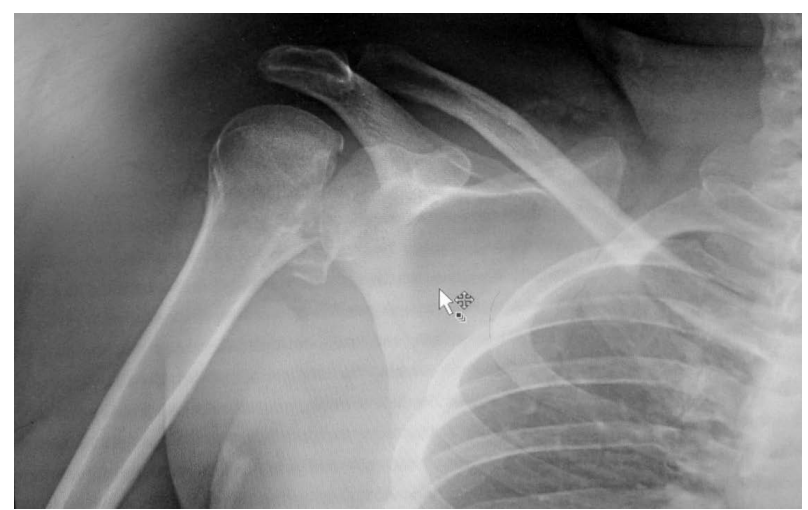

Male patient, 31 years old, trauma by fall from height, neglected posterior shoulder dislocation.

Figure 1. Preoperative plain X-ray with locking of humeral head.

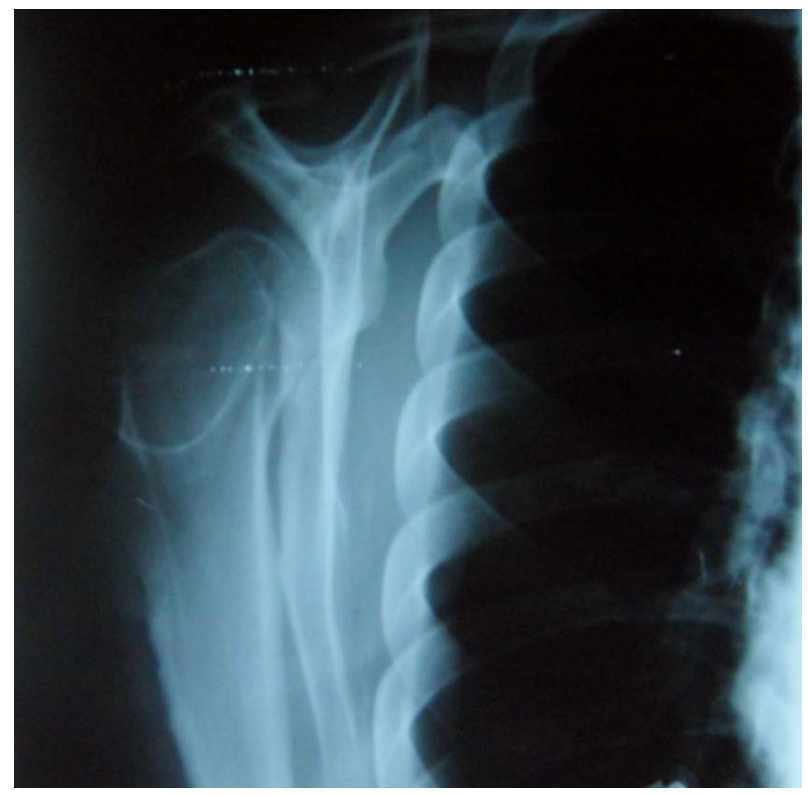

Figure 2. Preoperative plain X-ray scapular view.

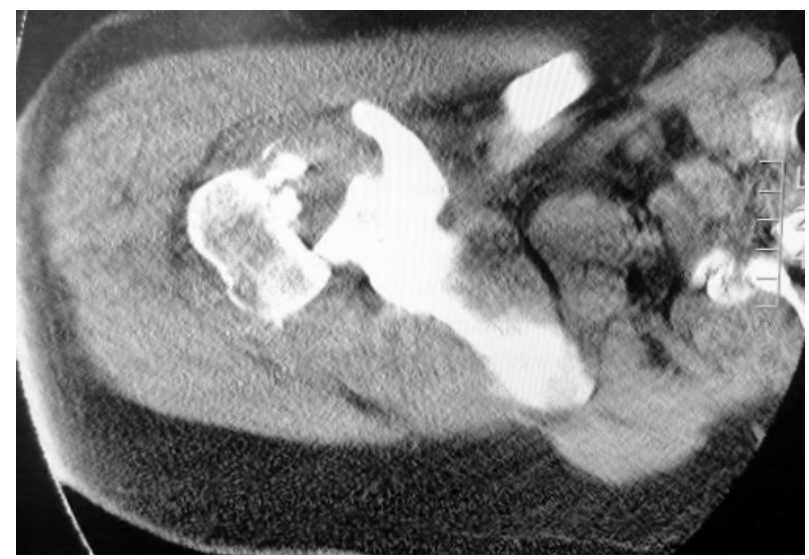

Figure 3. CT showing posterior shoulder dislocation with impingement of glenoid edge into humeral head. 
Table 1. Characteristics of patients.

\begin{tabular}{cccccccccc}
\hline Case No. & $\mathbf{1}$ & $\mathbf{2}$ & $\mathbf{3}$ & $\mathbf{4}$ & $\mathbf{5}$ & $\mathbf{6}$ & $\mathbf{7}$ & $\mathbf{8}$ & $\mathbf{9}$ \\
\hline Age & 30 & 25 & 28 & 46 & 41 & 22 & 27 & 31 & 25 \\
Gender & M & M & M & F & M & M & F & M & M \\
Side & Rt. & Rt. & Lt. & Lt. & Rt. & Rt. & Lt. & Rt. & Rt. \\
Trauma & - & FFH & Epi & - & FFH & Epi & Electr & FFH & FFH \\
Head defect \% & $\mathbf{4 5}$ & $\mathbf{3 6}$ & $\mathbf{3 8}$ & $\mathbf{4 1}$ & $\mathbf{3 8}$ & $\mathbf{4 3}$ & $\mathbf{4 0}$ & $\mathbf{4 0}$ & $\mathbf{3 9}$ \\
Follow-up & 20 & 17 & 18 & 20 & 14 & 18 & 16 & 18 & 21 \\
UCLA Pre-op. & 26 & 27 & 24 & 26 & 25 & 26 & 26 & 27 & 25 \\
\hline
\end{tabular}

$\mathrm{M}=$ male; $\mathrm{F}$ = female; Rt. = right; Lt. = left; FFH = fall from height; Epi. = Epilyptic fits; Electr. = Electric shock; Pre-op. = preoperative.

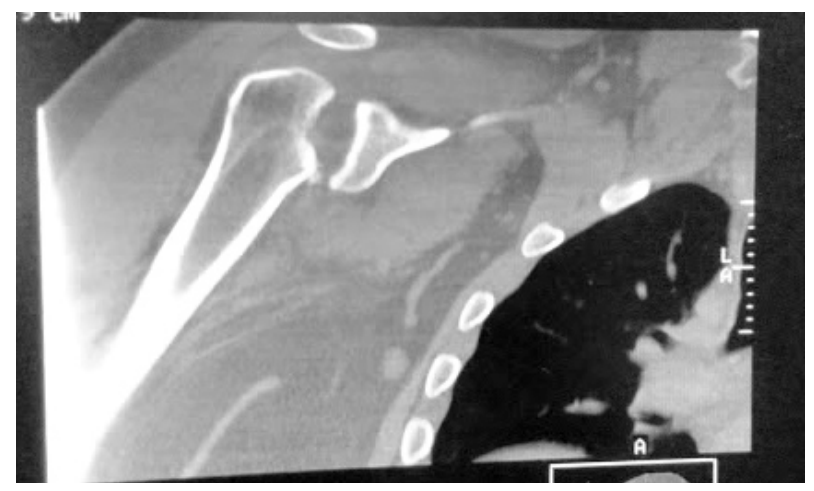

Reverse Hill-Sach (arrow)

Figure 4. CT Coronal cut demonstrating huge humeral defect.

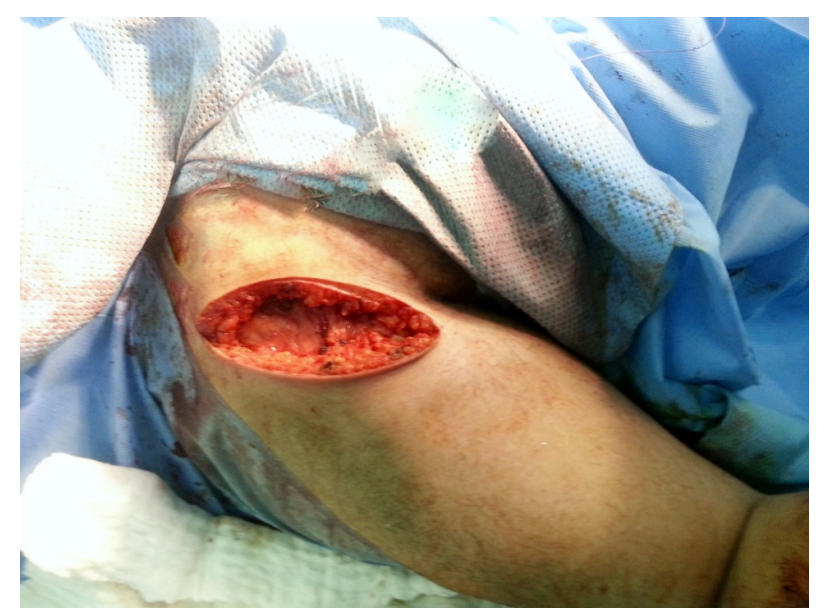

Figure 5. Delto-pectoral approach.

construct were evaluated intraoperatively by direct observation and fluoroscopy views through the entire range of motion. The wound was closed in layers using a suction drain.

Postoperatively, the shoulder was immobilized in $30^{\circ}$ abduction and neutral rotation for 4 weeks. During this period, the sling was removed for passive external rotation exercises only and internal rotation was not allowed during this period. A program of physical therapy initiated, including passive, active-assisted, and progressively active range of motion and rotator cuff strengthening exercises. At 12 weeks postoperatively, full activity was allowed. 


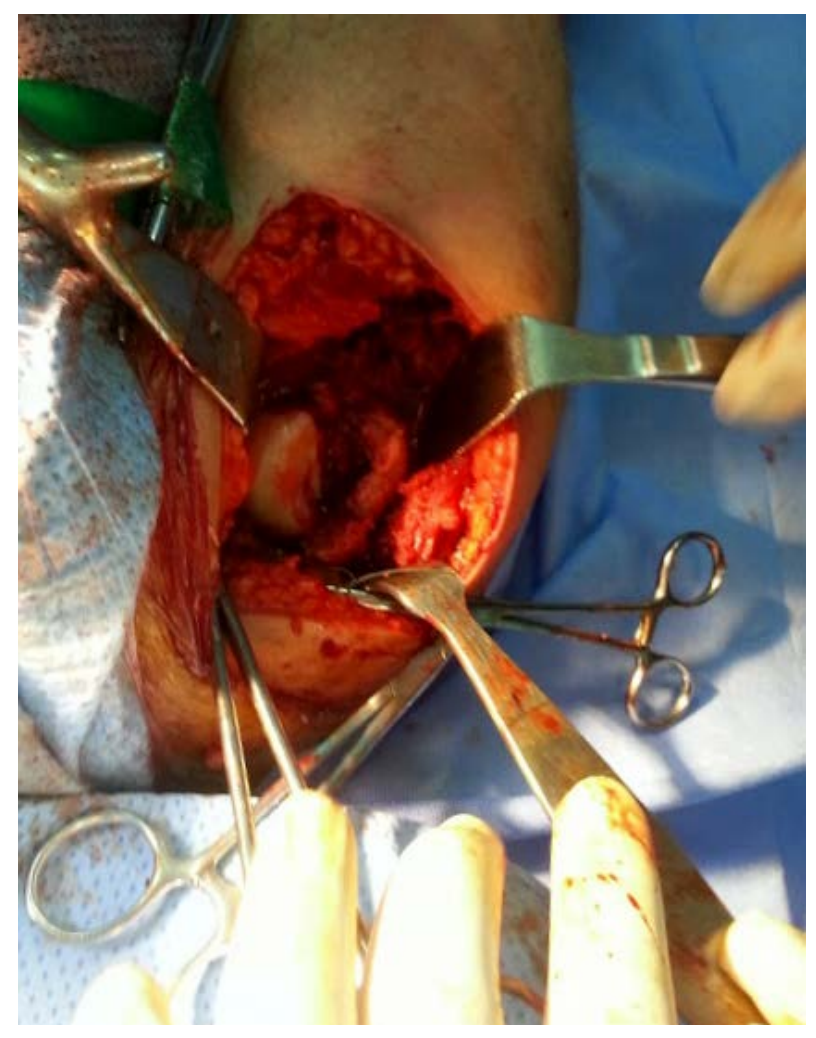

Figure 6. Intra-operative showing the humeral head defect.

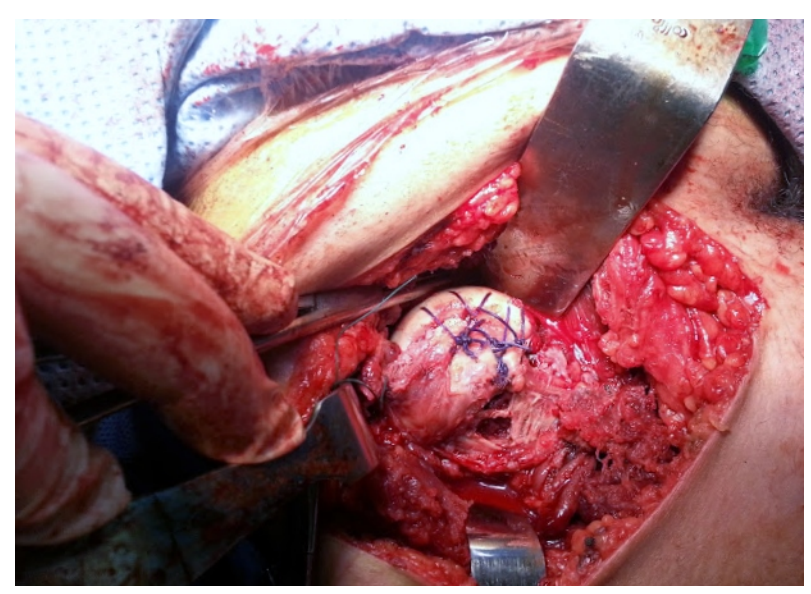

Figure 7. After transferring lesser tuberosity and suturing to humeral head via Ethibond [5] sutures.

\section{Results}

Total functional outcome was assessed using modified UCLA scoring system. Total UCLA scores immediate post-operatively averaged 25.7 (range, 21 - 27) out of 35. At last follow-up average UCLA scores had been improved to 31 (range, 27 - 34). All patients were satisfied with the level of function with no restrictions in activities of daily living. All patients were asymptomatic with painless and stable shoulder joints without apprehension or recurrence of instability. Mean range of shoulder motion was $166^{\circ}$ of forward flexion (range, $155^{\circ}-$ $175^{\circ}$ ), $75^{\circ}$ of external rotation (range, $60^{\circ}-85^{\circ}$ ), $50^{\circ}$ of internal rotation (range, $45^{\circ}-65^{\circ}$ ), and $155^{\circ}$ of abduction (range, $140^{\circ}-165^{\circ}$ ) (Figure 8 and Figure 9). The transferred tuberosity was incorporated completely in the humeral head defect within average 8 weeks (Figure 10). 


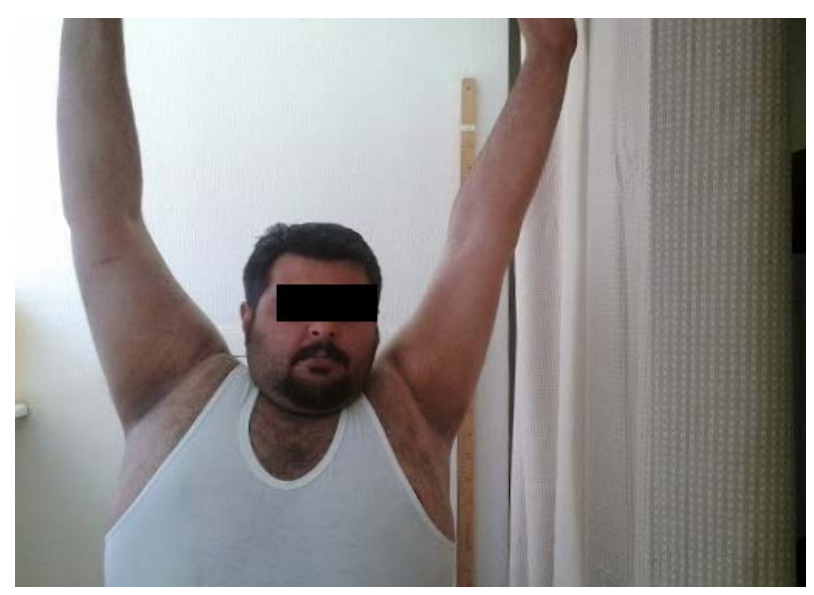

Figure 8. Patient 4 months post op. showing full forward flexion of shoulder.

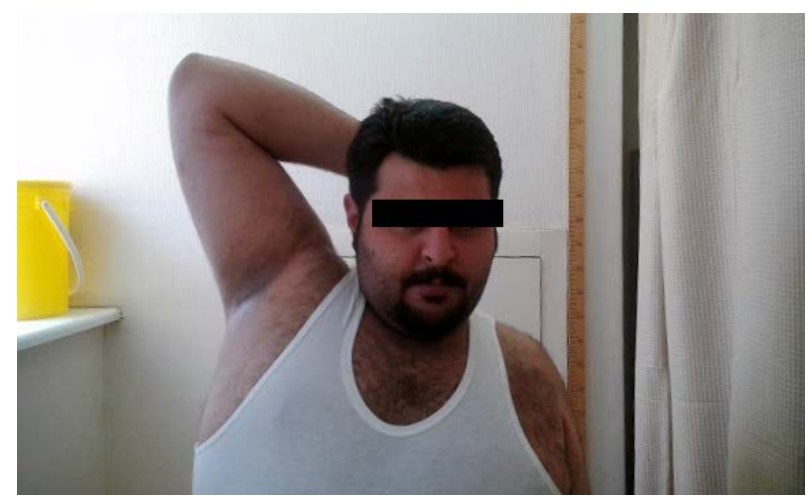

Figure 9. Full abduction external rotation.

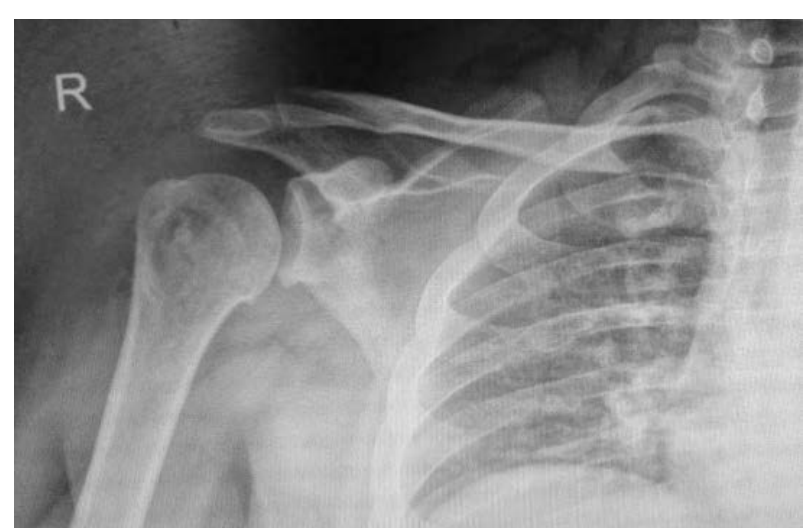

Figure 10. 4-month post-operative. The transferred tuberosity incorporated completely in the defect site within average 8 weeks.

On analyzing the functional outcome results at last follow-up, we get excellent results (30 - 35 points) achieved in 3 cases. Patients in this category have no pain; nearly normal shoulder motion; able to perform daily activities and even sports, adequate strength in lifting, pushing and throwing; with no shoulder instability. Good functional outcome achieved in 4 cases (26 - 30 points). Patients in this category have mild discomfort but no medications prescriped; they are capable of elevation, internal or external rotation of up to $75 \%$, mild to moderate limitation in daily activities and sports with no shoulder instability. Fair results achieved in one case (21 - 
25 points). The patients has moderate disabling pain, hence he is on occasional medications; capable of elevation, lacking internal or external rotation of up to $50 \%$; moderate limitation in overhead activities and lifting; unable to throw; mild-to-moderate apprehension of the arm in extended position. Poor results ( $<21$ points) achieved in one case. Patient has constant disabling pain, therefore constant medications prescribed; can barely reach the face; no rotation; unable to use the arm in useful activities and recurrent subluxation evident.

\section{Discussion}

Chronic locked posterior dislocation of the shoulder is an uncommon injury which has been unrecognized for more than three weeks. It is characterized by presence of an impression fracture of the articular surface of the humeral head [1]. The rarity of this injury, absence of clear radiographic signs in antero-posterior view unless an axillary view done and reluctance in clinical examination can lead to a missed diagnosis and delay of proper treatment. New technologies such as CT-scanning with 3D-reconstruction allow better visualisation and assessment of humeral head involvement and additional fracture lines [3]. Once this injury diagnosed, the management must be individualized depending on the amount of humeral head defect, the time passed from injury and degree of instability [10].

McLaughlin [5] described an operation in which he filled the defect with subscapularis tendon. The procedure was modified by taking a block of bone from the proximal humerus, including the lesser tuberosity and the attached subscapularis tendon and fixed it in the defect of the head with a screw. The transfer of lesser tuberosity instead of subscapularis alone was first introduced by Hawkins et al. [11]. The osteotomised bone fragment offers better filling of the defect and more secure reinsertion of the tendon [12]. The advantages of transfer of the lesser tuberosity are better bony filling of the humeral head and more secure reinsertion of the subscapularis tendon [13] (Figures 11-13). These techniques have been criticized in the literature because they change the humeral head anatomy. Also, the presence of hardware necessitates later removal [14]-[18]. In our protocol we used Ethibond suture number [5] and giant needle to pass tranosseous sutures to fix the lesser tuberosity to the main humeral head fragment. Accordingly, there is no need for second operation for hardware removal. Also, it is a cheap, less cost effect. Our study supports the use of the Neer and Hawkins modification of the McLaughlin procedure in patients with chronic, locked posterior dislocation of the shoulder. In spite of small number of cases in our series; the results are similar to those achieved by many authors utilizing subscapularis tendon and lesser tuberosity as a bone graft. We used a simpler $\&$ cheaper mode of fixation of the tuberosity graft which is suitable for underdeveloped countries.

\section{Conclusion}

This article described a modified McLaughlin technique using non-absorbable suture instead of the standard screw fixation for the lesser tuberosity graft in patients with neglected locked posterior dislocation of the shoulder.

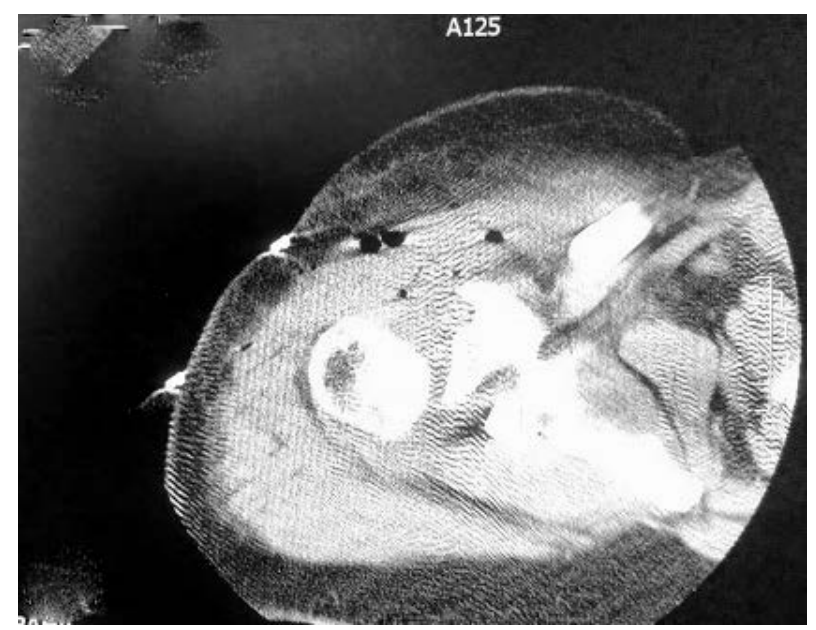

Figure 11. CT axial cut showing restoration of head contouring 4 months following surgery. 


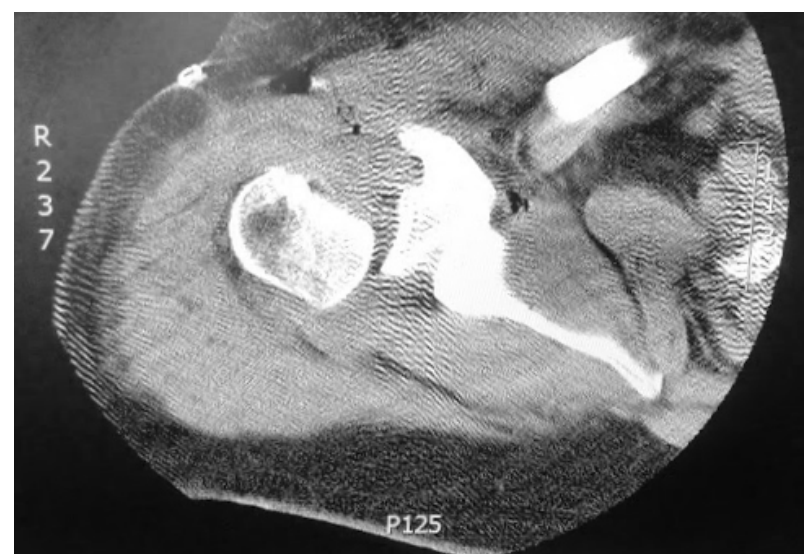

Figure 12. CT Axial cut showing restoration of head contouring at proximal cut.

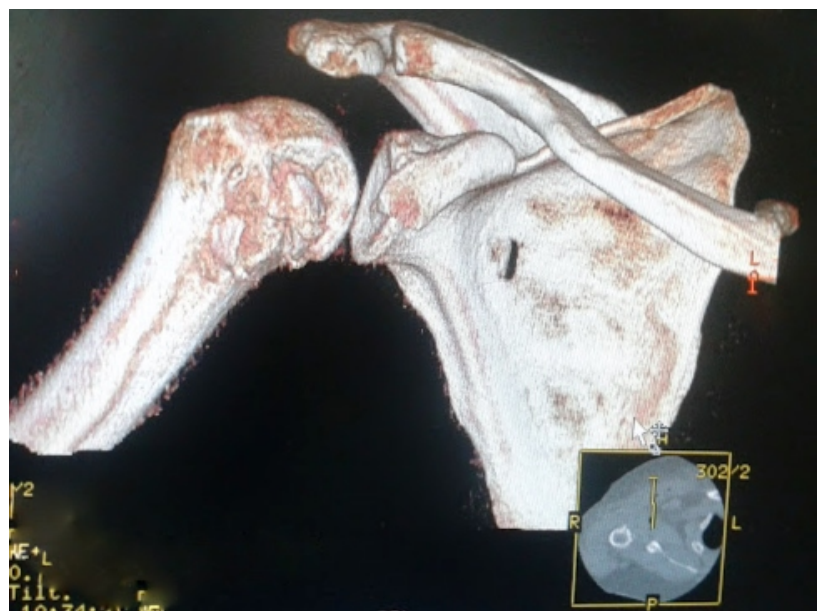

Figure 13. 3D CT 4 months showing transferred tuberosity within the defect.

Despite the delay in diagnosis and treatment, no head collapse or arthritis was observed at last follow-up in any patient. No hardware present which necessitates later removal and the technique is cheap which is suitable for underdeveloped countries. The grafts healed completely, and no recurrent instability occurred in any shoulder.

\section{Conflict of Interest}

The authors declare that they have no competing interests.

\section{Authors' Contributions}

Said K. revised the theoretical part of review, participated in the sequence alignment and drafted the manuscript. Ahmed Awad participated in the sequence alignment. M. Moazammi participated in the design of the study and performed the statistical analysis. Mohamed Adel conceived of the study, and participated in its design and coordination and helped to draft the manuscript. All authors read and approved the final manuscript.

\section{References}

[1] Constant, C.R. and Murley, A.H. (1987) A Clinical Method of Functional Assessment of the Shoulder. Clinical Orthopaedics and Related Research, 160-164.

[2] Checchia, S.L., Santos, P.D. and Miyazaki, A.N. (1998) Surgical Treatment of Acute and Chronic Posterior Fracture Dislocation of the Shoulder. Journal of Shoulder and Elbow Surgery, 7, 53-65. 
http://dx.doi.org/10.1016/S1058-2746(98)90183-5

[3] Wadlington, V.R., Hendrix, R.W. and Rogers, L.F. (1992) Computed Tomography of Posterior Fracture-Dislocations of the Shoulder: Case Reports. Journal of Trauma, 32, 113-115. http://dx.doi.org/10.1097/00005373-199201000-00024

[4] Aparicio, G., Calvo, E., Bonilla, L. and Espejo, L. (2000) Neglected Traumatic Posterior Dislocations of the Shoulder: Controversies on Indications for Treatment and New CT Scan Findings. Journal of Orthopaedic Science, 5, 37-42. http://dx.doi.org/10.1007/s007760050006

[5] McLaughlin, H.L. (1952) Posterior Dislocation of the Shoulder. Journal of Bone and Joint Surgery (America), 24, 584-590.

[6] Cicak, N. (2004) Posterior Dislocation of the Shoulder. Journal of Bone and Joint Surgery (British), 86, 324-332. http://dx.doi.org/10.1302/0301-620X.86B3.14985

[7] Keppler, P., Holz, U., Thielemann, F.W. and Meinig, R. (1994) Locked Posterior Dislocation of the Shoulder: Treatment Using Rotational Osteotomy of the Humerus. Journal of Orthopaedic Trauma, 8, 286-292. http://dx.doi.org/10.1097/00005131-199408000-00003

[8] Gerber, C. and Lambert, S.M. (1996) Allograft Reconstruction of Segmental Defects of the Humeral Head for the Treatment of Chronic Locked Posterior Dislocation of the Shoulder. Journal of Bone and Joint Surgery (America), 78, 376-382.

[9] Ozkan, M., Gul, O., Bacakoglu, K., Ozcan, C. and Ekin, A. (2000) Treatment of Posterior Locked Fracture Dislocation of the Shoulder with Hemiarthroplasty. Acta Orthopaedica et Traumatologica Turcica Journal, 34, 45-49.

[10] Sperling, J.W., Cofield, R.H. and Rowland, C.M. (2004) Minimum Fifteen-Years Follow-Up of Neer Hemiarthroplasty and Total Shoulder Arthroplasty in Patients Aged Fifty Years or Younger. Journal of Shoulder and Elbow Surgery, 13, 604-613. http://dx.doi.org/10.1016/j.jse.2004.03.013

[11] Hawkins, R.J., Neer, C.S., Pianta, R.M. and Mendoza, F.X. (1987) Locked Posterior Dislocation of the Shoulder. Journal of Bone and Joint Surgery, 69, 9-18.

[12] Robinson, C.M. (2005) Posterior Shoulder Dislocations and Fracture Dislocations. Journal of Bone and Joint Surgery (America), 87, 639-650.

[13] Randelli, M. and Gambrioli, P.L. (1986) Glenohumeral Osteometry by Computed Tomography in Normal and Unstable Shoulders. Clinical Orthopaedics and Related Research, 151-156.

[14] Loebenberg, M.I. and Cuomo, F. (2000) The Treatment of Chronic Anterior and Posterior Dislocations of the Glenohumeral Joint and Associated Articular Surface. Orthopedic Clinics of North America, 31, 23-34. http://dx.doi.org/10.1016/S0030-5898(05)70125-5

[15] Delcogliano, A., Caporaso, A., Chiossi, S. and Delcogliano, M. (2005) Surgical Management of Chronic, Unreduced Posterior Dislocation of the Shoulder. Knee Surgery, Sports Traumatology, Arthroscopy, 13, 151-155. http://dx.doi.org/10.1007/s00167-004-0524-6

[16] Robinson, C.M., Akhtar, A., Mitchell, M. and Beavis, C. (2007) Complex Posterior Fracture-Dislocation of the Shoulder. Epidemiology, Injury Patterns, and Results of Operative Treatment. Journal of Bone and Joint Surgery, 89, 14541466. http://dx.doi.org/10.2106/JBJS.F.01214

[17] Bock, P., Kluger, R. and Hintermann, B. (2007) Anatomical Reconstruction for Reverse Hill-Sachs Lesions after Posterior Locked Shoulder Dislocation Fracture. Archives of Orthopaedic and Trauma Surgery, 127, 543-548. http://dx.doi.org/10.1007/s00402-007-0359-y

[18] Charalambous, C.P., Gullett, T.K. and Ravenscroft, M.J. (2009) Modification of McLaughlin Procedure for Persistent Shoulder Instability: Technical Note. Archives of Orthopaedic and Trauma Surgery, 129, 753-755. http://dx.doi.org/10.1007/s00402-008-0721-8 